\title{
On the Efficiency of the Simple Groups of Order Less Than a Million and Their Covers
}

\author{
Colin M. Campbell, George Havas, Colin Ramsay, and Edmund F. Robertson
}

\section{CONTENTS}

1. Introduction

2. Methodology

3. Results

4. Commentary

5. Open Problems

Acknowledgments

References
2000 AMS Subject Classification: Primary 20-04, 20F05; Secondary 20D05, 20D06, 20D08

Keywords: Efficient presentations; simple groups; covering groups
There is much interest in finding short presentations for the finite simple groups. In a previous paper we produced nice efficient presentations for all small simple groups and for their covering groups. Here we extend those results from simple groups of order less than 100,000 up to order one million, but we leave one simple group and one covering group for which the efficiency question remains unresolved. We give presentations that are better than what was previously available, in terms of length and in terms of computational properties, in the process answering two previously unresolved problems about the efficiency of covering groups of simple groups. Our results are based on major amounts of computation. We make substantial use of systems for computational group theory and, in particular, of computer implementations of coset enumeration.

\section{INTRODUCTION}

There is much recent interest in finding short presentations for simple groups and their covering groups, witnessed by [Bray et al. 06, Campbell et al. 04, Guralnick et al. 07a, Guralnick et al. 07b, Korchagina and Lubotzky 06, Wilson 06]. This paper extends [Campbell et al. 04] to larger simple groups and relies on much material given there. We do not repeat the theoretical background information, but for completeness, we do provide an overview of the techniques and theorems used. We give the names of simple groups in Atlas format [Conway et al. 85], and we denote the covering group of the simple group $G$ by $\widehat{G}$. Various different measures for length of presentations appear in the literature. We use a simple one suitable for our purposes: the total length of the relators (after free and cyclical reduction) in the corresponding presentation.

Epstein studied geometric properties of groups in [Epstein 61]. He used homological arguments to show that there is a lower bound on the minimal number of relations required to present a group. He called a group efficient if this lower bound could be 
achieved. A relevant, more formal, description appears in [Campbell et al. 04].

A survey of results till 1989 for simple groups of order up to one million is given by Campbell, Robertson, and Williams in [Campbell et al. 89]. After this, progress was made starting in 2003 when $L_{3}(5)$ was shown to be efficient by Campbell, Havas, Hulpke, and Robertson [Campbell et al. 03] and $\widehat{M}_{22}$ was shown to be efficient in [Havas and Ramsay 03a], in both cases using techniques outlined here.

In the process of finding efficient presentations we answer two previously unresolved problems about the efficiency of covering groups of simple groups: we show that $\widehat{A}_{8}$ and $\widehat{L}_{2}(121)$ are efficient by giving efficient presentations for them. This leaves two questions about the efficiency of the simple groups of order less than one million and their covers to be answered. We address these and other unsolved problems in Section 5.

\section{METHODOLOGY}

We report on the results of systematic attempts to find efficient presentations of simple groups of order between $10^{5}$ and $10^{6}$ and of their covering groups. There are three distinct methods: we look at short presentations for perfect groups; we consider all essentially different generating pairs for simple groups; and we look at one-relator quotients of free products $C_{m} * C_{n}$ for coprime $m$ and $n$. These are the same kinds of techniques used in [Campbell et al. 04]. We outline them here and give references to more-detailed descriptions. We also state without proof the theoretical results that we use.

\subsection{Method 1}

A first source of efficient presentations for small simple groups and their covers is provided by censuses of short presentations of perfect groups, extending work in [Havas and Ramsay 03a]. The extension includes 2generator, 2-relator presentations with length up to 24; 2-generator, 3-relator presentations with length up to 26; 3-generator, 3-relator presentations with length up to 20; and selected one-relator quotients of $C_{m} * C_{n}$ (the free product of an $m$-cycle and an $n$-cycle) for coprime $m$ and $n$. (By a one-relator quotient of a particular group we mean a presentation obtained by adding one extra relator to a presentation for the specified group.) This last census overlaps with presentations considered by our third method.

\subsection{Method 2}

The second method uses a MAGma [Bosma et al. 97] program developed by Havas, Newman, and O'Brien [Havas et al. 04], which enables us to find all distinct generating sets for moderately sized permutation groups. (The program uses representatives from appropriately merged orbits of the action of the automorphism group of each permutation group studied.) We use this program to find such distinct generating pairs for groups under consideration, and then use the built-in algorithm of MAGMA to find a presentation of the group on some of these generating sets.

Presentations found in this way tend to have a reasonably small number of relators, increasing with increasing group order. They are rarely efficient, even for small groups. Often, however, simply checking all efficientsized subsets of the relators reveals efficient presentations. These checks are carried out by first quickly checking that a subset presents a perfect group (for otherwise it does not present a group we are seeking). Note that here we might be looking for either the underlying simple group or some stem extension. If this test is passed, then we attempt to check that the presentation is correct by coset enumeration; we generally use the ACE enumerator [Havas and Ramsay 01], either as available in GAP [GAP Group 04a] or MAGMA, or as a stand-alone program for some more-difficult cases.

\subsection{Method 3}

The third method has two variants. The first variant extends the idea of enumerating one-relator quotients of $C_{m} * C_{n}$ to building appropriate one-relator quotients that present a simple group, or a stem extension, by computing and testing relators that hold in the context of a specific generating set for a specific group. This is an easy modification of the process described by Campbell, Havas, Hulpke, and Robertson [Campbell et al. 03] using the GAP program PGRelFind [GAP Group 04b] and [Gamble et al. 04]. The second variant [Conder et al. 06] starts with the free product $C_{m} * C_{n}$ and its natural presentation $\left\{a, b \mid a^{m}, b^{n}\right\}$. We look for simple or perfect finite quotients of this group that can be obtained by adjoining a single extra relator. Thus we seek quotients of the form $\left\langle a, b \mid a^{m}, b^{n}, w(a, b)\right\rangle$, where $w=w(a, b)$ is a word in the generators $a$ and $b$ and their inverses $a^{-1}$ and $b^{-1}$, usually of relatively small length. We have implemented MAGMA programs that allow us to specify $m$, $n$, allowable lengths for $w$, and desired quotient groups. 


\subsection{Underlying Theory}

We use three results from [Campbell et al. 04] that enable us to amalgamate relators in presentations to give presentations for associated groups with fewer relators. The proofs of these results are constructive, which enables us to build efficient presentations using them.

Theorem 2.1. Let $G$ be a finite simple group. Suppose that $G$, or some stem extension of $G$, can be presented as

$$
\left\{a, b \mid a^{p}=b^{q}=w(a, b)=1\right\} .
$$

Then the covering group of $G$, all stem extensions of $G$, and $G$ itself are efficient.

Corollary 2.2. Let $G$ be a finite simple group. Suppose that $G$, or some stem extension of $G$, can be presented as

$$
\left\{a, b \mid u(a, b)^{p}=v(a, b)^{q}=w(a, b)=1\right\} .
$$

Suppose also that $u(a, b)$ and $v(a, b)$ generate the free group on $a$ and $b$. Then the covering group of $G$, all stem extensions of $G$, and $G$ itself are efficient.

Theorem 2.3. Let $G$ be a finite simple group. Suppose that $G$, or some stem extension of $G$, can be presented as

$$
\left\{a, b \mid u(a, b)^{p}=v(a, b)^{q}=w(a, b)=1\right\} .
$$

In addition suppose, for some integers $k$ and $l$, that $\widetilde{G}$ presented by

$$
\left\{a, b \mid u(a, b)^{k p} v(a, b)^{l q}=w(a, b)=1\right\}
$$

is perfect and generated by $u(a, b)$ and $v(a, b)$. Then $\widetilde{G}$ is the covering group of $G$.

\subsection{Effectiveness of Our Methods}

Each of our methods relies on investigations of search spaces of varying kinds. With increasing group size and presentation length the search spaces grow enormously. In [Campbell et al. 04], where the underlying simple groups have size less than $10^{5}$, we give some graphic examples of this.

Such limitations become more pronounced with the larger groups considered here. We give some rough CPU times taken for some of the lengthier computations. The CPU times are for runs on a Sun SPARC V9 machine with a $750-\mathrm{MHz}$ CPU running SunOS. For example, in some cases partial searches with Method 2 ran for several CPU months.
Each of our methods is impacted by this, and so in contrast to the situation in [Campbell et al. 04], where we generally applied each of the three methods to every group, here we usually did not further study a simple group in great detail once we had found an efficient presentation for it and its cover. What this means is that we applied Method 1, which is based on searching among presentations for perfect groups, for all groups in this catalogue. However we did not necessarily try Method 2 or 3 .

Sometimes we have not succeeded in using our methods to directly find efficient presentations for simple groups. Instead we first found efficient presentations for a covering group, from which we could then readily produce longer, but still efficient, presentations for the simple group by factoring out the center of the covering group.

One problem we face in producing our table of new presentations is that once we are able to find an efficient presentation for a group, then there are arbitrarily many. We say that two presentations are equivalent if the relations of each hold in the groups defined by them. In Table 2 we give an instance of a presentation with shortest-found length. We give coset enumeration performance measured by the total number of cosets used in a successful enumeration of the trivial subgroup using the Hard strategy of ACE, with the group generators given in alphabetical order.

We take the presentation as produced by our process and generally do not make major efforts like those described in [Havas and Ramsay 00] to improve it. Thus we give presentations produced by MAGMA at most slightly simplified, but with various relators in what may seem less-natural forms (in the sense that inversion or cyclic rotation may produce more-usual forms). In contrast, presentations from censuses of short presentations arise with relators in canonical form, as described in [Havas and Ramsay 03a]. Generally speaking, there are often other variants, including longer presentations, that enumerate better. We comment on each of the groups in Section 4.

As far as reliability of results is concerned, we assert that all presentations given in this paper correctly define the groups. Each new presentation that appears has been verified by both GAP and MAGMA programs to present the specified group. Here we intentionally do not use ACE for the GAP check, but rather use GAP's internal coset enumerator (which was entirely independently written), providing a greater level of confidence in the results. 


\begin{tabular}{llrr}
\hline Name & Relators and/or Relations & Length & Cosets \\
\hline$U_{3}(5)$ & $x^{2}=(x y)^{10}, x y^{-2} x y^{2} x\left(y x y^{-2} x y x y\right)^{2},\left(x, y^{2}\right)^{4}=y^{5}$ & 68 & 197015911 \\
$\widehat{U}_{3}(5)$ & not known to be efficient & & \\
$J_{1}$ & $a^{2} b^{3},\left(A B(a b)^{2}\right)^{4}(A b)^{2} A B(a b)^{3} a B(a b)^{2}(A B)^{2}$ & 51 & 8187235 \\
$A_{9}$ & $x y X y x Y, x^{15}, x y\left(x y^{2}\right)^{2}\left(x^{2} y\right)^{2} x^{2}\left(x^{2} y^{2}\right)^{2}$ & 45 & 944000 \\
$\widehat{A}_{9}$ & not known to be efficient & & \\
$L_{2}(64)$ & $x y X y x Y, x^{4} y x y^{4} x^{5} y^{5} x^{5} y^{4} x y$ & 37 & 5102529 \\
$L_{2}(81)$ & $a^{2}, b^{3}, a b\left(a B(a b)^{3}\right)^{2}(a B)^{4}\left((a b)^{3} a B\right)^{2}$ & 47 & 412909 \\
$\widehat{L}_{2}(81)$ & $a^{2} b^{3}, a b A B(A b)^{3} A B(a b)^{3}(A B)^{4}\left((a b)^{3} a B\right)^{2}$ & 47 & 2172625 \\
$L_{3}(5)$ & not known to be efficient & & \\
$M_{22}$ & $a^{2}=(a b)^{11},\left(a b a b^{2}\right)^{7}=b^{4}$, & & \\
& $(a b)^{2}(a B)^{2} a b^{2}(a b)^{2} a B a b\left(a b^{2}\right)^{2}=b^{4}$ & & \\
$\widehat{M}_{22}$ & not known to be efficient & 43 & 607059999 \\
$J_{2}$ & $a^{2}, b^{3},(a b)^{2}(a B)^{2} a b(a B)^{4}(a b)^{3}(a B)^{4} a b(a B)^{2}$ & 43 & 1469303 \\
$\widehat{J}_{2}$ & $a^{2} b^{3},(a b)^{2}(a B)^{2} a b(A B)^{4}(A b)^{3}(A B)^{4} a b(a B)^{2}$ & 61 & 3672993 \\
$L_{2}(121)$ & $a^{3}=b^{11},\left(a b^{4} a b^{7}\right)^{2}, a B^{2} A b^{3} a b^{2} A b^{3} a B^{2} A B^{3}$ & 61 & \\
$\widehat{L}_{2}(121)$ & not known to be efficient & & \\
$L_{2}(125)$ & $a^{2}, b^{3},(a b)^{4}(a B)^{14}(a b)^{4}(a B)^{-7}$ & 63 & 5389408 \\
$\widehat{L}_{2}(125)$ & $a^{2}=b^{3},(A b)^{4}(a B)^{14}(A b)^{4}(a B)^{-7}$ & 63 & 22877752 \\
$S_{4}(4)$ & not known to be efficient & & \\
\hline
\end{tabular}

TABLE 1. Shortest efficient presentations as of 1989 .

\begin{tabular}{llllll} 
Name & Relators & Length & Cosets & Order & Ref \\
\hline$U_{3}(5)$ & $a^{7}, a b A b b a b A B B B, a a b A B B a B a b a b$ & 30 & 313132 & 126000 & 4.11 \\
$\widehat{U}_{3}(5)$ & not known to be efficient & & & 378000 & 4.11 \\
$J_{1}$ & $x y X y x Y, x^{3}(y x)^{2} x y^{2} x^{3} y^{3} x y x^{2} y^{3}$ & 29 & 1372504 & 175560 & 4.1 \\
$A_{9}$ & $a^{4}, a B a b A B A B B A b b, a b A B a B a B A B a a B$ & 29 & 291299 & 181440 & 4.2 \\
$\widehat{A}_{9}$ & $x y X y x Y, x^{3} y^{3} x Y^{2} X^{2} Y(x y)^{3} y x(x y)^{3} y x y$ & 35 & 72204121 & 362880 & 4.2 \\
$L_{2}(64)$ & $a a b A B a B A b, a a b^{3} a a B^{4}$ & 20 & 6046093 & 262080 & 4.3 \\
$L_{2}(81)$ & $(x Y)^{3},(y X y)^{2}, x^{4} y^{3} x y^{3} x y x y^{3} x y^{3}$ & 33 & 385827 & 265680 & 4.4 \\
$\widehat{L}_{2}(81)$ & $a a b A B A b, a^{3} B a b b A A b b A A b b a B$ & 24 & 912581 & 531360 & 4.4 \\
$L_{3}(5)$ & $x y X y x Y, x^{4}\left(y^{2} x\right)^{2} x^{2} y^{3} X^{3} Y X(y x x)^{2} y$ & 33 & 63268192 & 372000 & 4.5 \\
$M_{22}$ & $a^{5}(A b)^{3}, a a b A B a b b A B, b^{11}$ & 30 & 2104858 & 443520 & 4.6 \\
$\widehat{M}_{22}$ & $a a b a b A A B, a b b b b a B a B$ & 17 & 21611026 & 5322240 & 4.6 \\
$J_{2}$ & $a^{3}, b^{5}, a b a b b A B A b b a b a B$ & 19 & 1211899 & 604800 & 4.7 \\
$\widehat{J}_{2}$ & $a a b a a B A A B, a b b b a B B a B B$ & 2972373 & 1209600 & 4.7 \\
$L_{2}(121)$ & $(x Y)^{3},(y X y)^{2}, x^{10} y^{2} x^{4} y^{3} x^{4} y^{2}$ & 22 & 3732563 & 885720 & 4.8 \\
$\widehat{L}_{2}(121)$ & $a^{4} b A A B A A b, a^{3} b A B^{4} A b$ & 35 & 1327887 & 976500 & 4.9 \\
$L_{2}(125)$ & $(x Y)^{3},(y X y)^{2}, x^{7} y x^{2} y x y x y^{2} x y x y x^{2} y$ & 35084 & 1771440 & 4.8 \\
$\widehat{L}_{2}(125)$ & $a^{3} b A A B A A b, a^{3} B A B a^{3} B^{4}$ & 23 & 2852237 & 1953000 & 4.9 \\
$S_{4}(4)$ & not known to be efficient & & & 979200 & 4.10 \\
\hline
\end{tabular}

TABLE 2. Our short presentations. 


\section{RESULTS}

We give nice efficient presentations for simple groups of order between $10^{5}$ and $10^{6}$ and for their covering groups. We exclude $L_{2}(p)$ for prime $p \geq 5$ (for which [Sunday 72] gives efficient presentations) and $\widehat{L}_{2}(p)$ (for which [Campbell and Robertson 80] gives efficient presentations). We also exclude $S_{4}(4)$ (the smallest simple group for which no efficient presentation is known) and $\widehat{U}_{3}(5)$ (the smallest covering group for which no efficient presentation is known).

We put our efficient presentations in context by comparing them with the shortest presentations known in 1989, before the availability of the methods outlined in Section 2. Sometimes those presentations imply the existence of shorter presentations. For example, where presentations include relators like $a^{2}$ and $b^{3}$ or like $a^{2} b^{3}$, it is possible to follow the ideas explained in [Campbell et al. $86]$ to give shorter presentations on $x=a b$ and $y=a B$ or $y=A B$.

We adopt the convention of using uppercase letters to denote inverses in presentations, so that, for example, $A=a^{-1}$. We give presentations by listing sets of relators and/or relations (often only implicitly specifying the generators). For coset enumeration purposes the generators are always given in alphabetical order.

The tables (which have the simple groups listed in order of increasing size) give the name of the simple group, relators and/or relations for the group, the total length of the relators in the corresponding presentation (freely and cyclically reducing relators as done by ACE), and the total number of cosets used in a successful coset enumeration for this presentation over the trivial subgroup using the Hard strategy of the ACE enumerator. The presentations in Table 1 appear in [Jamali and Robertson 89, Campbell and Robertson 88, Campbell et al. 86, Campbell et al. 90], or [Campbell et al. 89]. In Table 2 we provide a reference to the subsection in which we give further information on the group.

\section{COMMENTARY}

Our methodology produces a very large number of efficient presentations for most of the groups under consideration. Then simple modifications to these lead to many more presentations that are efficient. In our tables we have given, from among the shortest efficient presentations that arose as one of our generated presentations, one that enumerates with the fewest number of total cosets over the trivial subgroup. (We emphasize that we use this purely as a measure of coset enumeration performance and do not suggest that enumerations over the trivial subgroup are the best way to compute with the presentation to gain other information about the group.) We also give further information for each simple group.

The ordering of the subsections in this section is a little unusual. It might seem natural to put the groups in order of increasing size of simple group. We have done this with one exception: we have put the smallest, $U_{3}(5)$, last because it leads to a problem that we have not solved and it makes more sense to consider it after the others.

\section{$4.1 \quad J_{1}$}

The presentation for $J_{1}$ in Table 2 was obtained by applying Corollary 2.2 to a one-relator quotient of $C_{2} * C_{3}$, with the relator found by an intelligent search. A predecessor of our presentation arose in Method-1 censuses, in addition to one other inequivalent presentation. The instances that we use started with the two relators $(x Y)^{3}$ and $(y X y)^{2}$, to which we added $w_{1}=x^{3} y x y x x y y x^{3} y^{3} x y x x y^{3}$ and $w_{2}=$ $x^{4} y x^{3} y x x y^{3} x x y x x y x x y$, respectively. Coset enumerations of the 175560 cosets over the trivial subgroup for these 3-relator presentations took totals of 554222 and 2674513 cosets, respectively. We use $w_{1}$ in our tabulated presentation. (The analogous presentation with $w_{2}$ uses 11761688 cosets.)

Further, a study of one-relator quotients of $\left\{a^{3}, b^{7}\right\}$ yields four inequivalent length-14 words that may be added to give presentations of $J_{1}$, namely

$$
\begin{aligned}
& \text { ababababAbaBaB, abababaBaBabAb, } \\
& \text { ababaBaBaBaBAB, ababaBABaBaBaB.}
\end{aligned}
$$

Presentations using these relators take 322550, 305976, 370801 , and 318374 total cosets over the trivial subgroup, respectively. The proof of Theorem 2.1 in [Campbell et al. 04] explains how to convert these presentations into efficient ones for $J_{1}$, longer than our tabulated one.

The group $J_{1}$ has 88570 distinct generating pairs, and we looked at 7982 of these (in a computation taking two CPU months), yielding 554 3-relator presentations. Among those were some that could be processed using the ideas in the proofs of Theorem 2.1 or Corollary 2.2 to give other efficient presentations for $J_{1}$. These include $\left\{b^{3}, a B A A B a a b a b a b,(B a)^{7}\right\},\left\{b^{2}\right.$, $\left.a^{3} b A^{3} b a a b A A b A^{4} b a^{3} b A A b a^{3} b a^{3} b a,(b A)^{3}\right\},\left\{b^{7},(B A)^{3}\right.$, $\left.a B A^{5} B A A B a b\right\}$, and $\left\{b a b A B A B a b^{3} a b a b,(a b a)^{2}, a^{7}\right\}$. 


\section{2 $\quad A_{9}$}

Neither $A_{9}$ nor $\widehat{A}_{9}$ arose in our Method-1 censuses. We explain how we used Methods 2 and 3 to find efficient presentations for them.

The group $A_{9}$ has 39247 distinct generating pairs and $\widehat{A}_{9}$ has 155118 . We investigated distinct generating pairs of $A_{9}$ for $24 \mathrm{CPU}$ days, during which time we looked at 3861 pairs. In the process we found 107 efficient presentations for $A_{9}$, with lengths between 29 and 57. There was only one presentation with length 29 , so we tabulate it.

None of the 107 presentations was of a form suitable for the application of Theorem 2.1, Corollary 2.2, or Theorem 2.3. This meant that we were not able to use any of them to build an efficient presentation for $\widehat{A}_{9}$. We investigated 4855 of the distinct generating sets for the cover (in a computation taking two CPU months) and failed to find any efficient presentations or any presentations to which Theorem 2.1, Corollary 2.2, or Theorem 2.3 could be applied.

In the end, $\widehat{A}_{9}$ succumbed to Method 3 using PGRelFind (in a run taking $84 \mathrm{CPU}$ hours). We studied longer one-relator quotients of $C_{2} * C_{3}$. On mapping $C_{2} * C_{3}$ onto a representation of $A_{9}$ satisfying presentation 16.4 of [Campbell and Robertson 84], we found various words with length greater than or equal to 54 that mapped onto the identity. There were seven words with length 54 produced, which give equivalent presentations. For all of these we could show that they suffice to produce $\widehat{A}_{9}$ as a one-relator quotient of $C_{2} * C_{3}$.

Starting with $\left\{a, b \mid a^{2}, b^{3}\right\}$ we found, inter alia, that the word

\section{$w=a b a b a B a b a b a b a b a B a B a B a b a B a b a b a$ BabaBabaBaBababaBabaBabab}

maps to the identity in the representation of $A_{9}$. We investigated the group presented by $\left\{a, b \mid a^{2}, b^{3}, w\right\}$ and found (by coset enumeration) that it is $\widehat{A}_{9}$, using 38120066 cosets over the trivial subgroup. The proof of Theorem 2.1 then delivers us a very large number of efficient presentations for $\widehat{A}_{9}$ with length 59 and larger.

One way to obtain length-59 presentations is to take the word $w$ and construct $\widetilde{w}$ from $w$ by replacing the sequence of $a$ 's in $w$ by any one of the sequences generated from a multiset with $15 \mathrm{a}$ 's and $12 \mathrm{~A}$ 's. There are clearly $\left(\begin{array}{l}27 \\ 12\end{array}\right)=17383860$ such sequences, which lead to that number of superficially different (but equivalent) presentations $\left\{a^{2} b^{3}, \widetilde{w}\right\}$ for $\widehat{A}_{9}$.

Now defining $x=a b$ and $y=a B$, so that $a=x Y x$ and $b=X y$, we obtain presentations on $x$ and $y$. We did this for all $\left(\begin{array}{l}27 \\ 12\end{array}\right)$ presentations for $\widehat{A}_{9}$ obtained as described above and simplified them. This led to presentations with lengths varying from 35 to 91 . The full length distribution that we obtained this way was as follows (length, number of presentations):

35,$3544 ; 37,97361 ; 39,663083 ; 41,1538547$;

43, 1980852; 45, 2334578; 47, 2365991; 49, 2114241;

51,$1744472 ; 53,1397000 ; 55,1066256 ;$ 57, 768446;

59,$502991 ; 61,332949 ; 63,194842 ; 65,132423$;

67,$67975 ; 69,39145 ; 71,19279 ; 73,9724 ; 75,6136$;

77,$2128 ; 79,1124 ; 81,468 ; 83,158 ; 85,112 ; 87,18$;

89,$12 ; 91,5$.

This shows how dramatically the number of efficient presentations for a group may grow. We simply list the canonical version of the first of the length-35 presentations for $\widehat{A}_{9}$ that we found.

\section{$4.3 \quad L_{2}(64)$}

For $L_{2}(64)$ we tabulate a length-20 presentation from a Method-1 census of short 2-generator, 2-relator presentations for perfect groups. It is interesting to note that it is one shorter than the shortest presentation that we have obtained [Campbell et al. 04] for $L_{2}(32)$. It is the unique shortest canonical 2-relator presentation for this group that we have found. The census of 3-generator, 3relator presentations with length up to 20 revealed nine length-20 presentations for this group, varying only in their longest relator. The one that enumerates best, using 629376 cosets, is $\{a a b A B, b c b c B c, a b c a c c c c b\}$.

We have found 2-generator, 2-relator presentations that are longer but better for coset enumeration than our Table 2 presentation. At length 23 we have

$$
\left\{a^{3} b A B A b, a^{3} B B A A b A A b A A B B\right\},
$$

which uses 924002 cosets, and at length 24 we have

$$
\left\{a a b a a B A A B, a^{5} b a B a B B a a B B\right\},
$$

which uses 398265 .

The shortest presentations, length 24, that we found that can be readily seen as consequences of the application of Corollary 2.2 include

$$
\left\{a^{7}\left(A^{2} b\right)^{2}, a a b b a B a B^{3} A b A b b\right\}
$$

and

$$
\left\{a^{5}(A b)^{2}, a b A B^{3} A b a b b a B B a b b\right\},
$$

which use 1441467 and 12173611 cosets, respectively. A representative of the shortest presentations that we have found for $L_{2}(64)$ as a one-relator quotient of $C_{2} * C_{3}$ is

$$
\left\{(x Y)^{3},(y X y)^{2}, x^{5} y x^{3} y x y^{4} x y x^{3} y\right\},
$$

which uses 314212 cosets. 


\section{$4.4 \quad L_{2}(81)$}

The presentation in Table 2 for $L_{2}(81)$ is a one-relator quotient of $C_{2} * C_{3}$. In our Method-1 censuses of such quotients we found two essentially different presentations for this group with long relator having length 21; the other one is

$$
\left\{(x Y)^{3},(y X y)^{2},\left(x^{2} y\right)^{2} x y^{5} x^{2} y^{5} x y\right\},
$$

which uses more cosets, 1861721.

For $\widehat{L}_{2}(81)$ we tabulate a presentation from a Method1 census of short 2-generator, 2-relator presentations. It is the unique shortest canonical 2-relator presentation for this group that we have found. Longer presentations can be constructed from our presentations for $L_{2}(81)$.

\section{$4.5 \quad L_{3}(5)$}

The efficiency problem for $L_{3}(5)$ was resolved in [Campbell et al. 03]. There the presentation

$$
\begin{gathered}
\left\{a^{2} b^{3}, \quad A b A b A B A b A b A B a B a B A b A b A B A b A b\right. \\
A B a B a B a B a b a B a B a b a B a B A B A B\}
\end{gathered}
$$

was revealed. It was derived from presentation 17.6 of [Campbell and Robertson 84]. It has length 55 and uses a total of 92162535 cosets. Equivalents of this presentation were revealed by our censuses. Then, via the same kind of transformation to generators $x$ and $y$ as used above for $\widehat{A}_{9}$, we looked at $\left(\begin{array}{l}25 \\ 11\end{array}\right)$ presentations, revealing 4457400 presentations with lengths from 33 to 85, including 1651 with length 33 . We list the canonical version of the first of the length-33 presentations for $L_{3}(5)$ that we found.

Our censuses also revealed a longer (length-41) presentation that gives $L_{3}(5)$ as an explicit one-relator quotient of $C_{2} * C_{3}$ :

$$
\left\{a^{3}(a b)^{2}, a b b a B B a B^{4} a b^{3} A b b A B^{3} a b^{3} A b^{3} a B^{3}\right\} .
$$

This presentation is harder for coset enumeration, using 237949556 cosets.

\section{$4.6 \quad M_{22}$}

The group $M_{22}$ and its covering group are studied in detail in [Conder et al. 06], motivated in part by the fact that $\widehat{M}_{22}$ has surprisingly short efficient presentations. Here we simply list the shortest efficient presentations for these groups revealed in that paper, which contains comprehensive information about efficient presentations for both groups. Briefly, the presentation for $\widehat{M}_{22}$ is a shortest presentation possible, while the presentation for $M_{22}$ is built by adding a relator that kills the center in the context of a slightly longer presentation of $\widehat{M}_{22}$.

\section{$4.7 \quad J_{2}$}

Two inequivalent efficient presentations for $J_{2}$ as onerelator quotients of $C_{2} * C_{3}$ appear in [Campbell and Robertson 84] and [Campbell and Robertson 88]. Our censuses revealed equivalents of these. We also found other efficient presentations as one-relator quotients of $C_{3} * C_{5}, C_{2} * C_{7}$, and $C_{3} * C_{10}$. We tabulate the shortest we found, a one-relator quotient of $C_{3} * C_{5}$. A presentation longer by 1 , namely $\left\{a^{5},(a b)^{3}\right.$, aabbaa $\left.B B A A B B\right\}$, enumerates better ( 837516 cosets).

For $\widehat{J}_{2}$ we tabulate a presentation from a census of short two-relator presentations for perfect groups that is the unique shortest canonical two-relator presentation for this group. Very many efficient presentations arise from the various presentations for $J_{2}$ as a one-relator quotient of $C_{m} * C_{n}$. These include $\left\{x y X y x Y, x^{3} y^{4} x y y x^{3} Y X Y X X Y x y\right\}$, which may be derived from a one-relator quotient of $C_{2} * C_{3}$ and uses 1375550 cosets.

\section{8 $\quad L_{2}(121)$}

The presentation in Table 2 for $L_{2}(121)$ is a one-relator quotient of $C_{2} * C_{3}$. In our Method-1 censuses of such quotients we found three inequivalent presentations for this group with long relator having length 25; the others are

$$
\left\{(x Y)^{3},(y X y)^{2}, x^{5} y^{2} x y x\left(x y^{2}\right)^{3} x^{2} y x y^{2}\right\}
$$

and

$$
\left\{(x Y)^{3},(y X y)^{2}, x^{3} y x y^{3} x y x^{3} y^{3} x y^{4} x y^{3}\right\},
$$

which use more cosets, 4619952 and 72687164, respectively.

For $\widehat{L}_{2}(121)$ we tabulate a presentation from a Method-1 census of short 2-generator, 2-relator presentations. It is the unique shortest canonical 2-relator presentation for this group that we have found. Longer presentations can be constructed from our presentations for $L_{2}(121)$.

\section{$4.9 \quad L_{2}(125)$}

The presentation in Table 2 for $L_{2}(125)$ is a one-relator quotient of $C_{2} * C_{3}$. Up to equivalence, it is the only presentation with this length that we have found.

For $\widehat{L}_{2}(125)$ we tabulate a presentation from a Method-1 census of short 2-generator, 2-relator presentations. It is the unique shortest canonical 2-relator presentation for this group that we have found. Longer presentations can be constructed from our presentation for $L_{2}(125)$. 


\begin{tabular}{lrr} 
Relators & Length & Cosets \\
\hline$b a B a^{3} B a b b,(a b A A B a)^{2}, a B a B A^{3} b b A^{3} B$ & 35 & 2464234 \\
$(a B B)^{2}, \quad A b a a b a^{3} b a a b A B, \quad A B B A B A^{3} B^{3} A^{3} B$ & 35 & 3786512 \\
$(a b a a)^{2}, \quad b A A B a a b a b b, \quad A B A b a B a B A A B a B a b A B A B$ & 37 & 2492551 \\
$(a B B a)^{2}, \quad b A A B a a b a b b, A B A b a B a B A A B a B a b A B A B$ & 37 & 4191954 \\
$\left(a B^{4}\right)^{2}, a b a B B A B A b b A B A B B, \quad A b^{3} A B A A B B A B a b A$ & 40 & 2480214 \\
$\left(a B^{4}\right)^{2}, a b a B B A B A b b A B A B B, \quad b a B A B B A A B A b^{3} A A$ & 40 & 2534585 \\
$(a B A b a)^{2}, B a B a a B A A B a b^{3} A A, B^{3} a B B A B a b b A B B A B$ & 41 & 3605111 \\
$b A A B a a b a b b, A B a a B A A B B A B B A, \quad A B A b a B a B A A B a B a b A B A B$ & 42 & 1077562 \\
$a^{2}, B^{4} a B^{4} a B a B^{3} a B a, b a b b a B^{6} a b b a b a b a B a b a$ & 43 & 7036654 \\
$a^{6}, a B a B a a B a b b, \quad b b b A A A B a a a B B a a, b^{17}$ & 47 & 1041750 \\
$(a b)^{2}, a B(B a a)^{2} b b a a B a, a^{17}, A B B a a B A^{3} b A b A^{4} b b a B a^{4} B a B a^{3} b A$ & 67 & 2657240 \\
$(a b)^{2}, A B a B B a^{4} b b a B A^{3}, a^{17}, A B B a a B A^{3} b A b A^{4} b b a B a^{4} B a B a^{3} b A$ & 69 & 1428848 \\
$b^{4}, A B B a b A b A A b b a b b a b A b, a B B A b A B B A A b b A b A b b a B, \quad(a b)^{17}$ & 75 & 9061870 \\
\hline
\end{tabular}

TABLE 3. Interesting presentations for $S_{4}(4)$.

\section{$4.10 \quad S_{4}(4)$}

A substantial computational effort aimed at finding an efficient presentation for $S_{4}(4)$ was already made in [Campbell et al. 03]. This led to a presentation with one more relator than an efficient one. We tried each of Methods 1,2 , and 3 but failed to obtain an efficient presentation. Methods 1 and 3 are hampered by the fact that $S_{4}(4)$ is not generated by any pair of elements of which one is an involution and the other has order 3. Using Method 2 we found 117843 distinct generating sets and looked at only some of them. (It took 19 CPU hours to find the generating sets, then over $2 \mathrm{CPU}$ hours, on average, to construct a presentation on a given generating set.)

The presentations started off looking quite unwieldy; the average number of relators was 16 . Indeed, we varied the method to look at a random sample of about 10000 generating sets. In a computation taking $36 \mathrm{CPU}$ days we did find many shorter presentations with one extra relator, including those in Table 3.

We also looked at some two-relator quotients of $C_{m} *$ $C_{n}$ and found four that present $S_{4}(4)$. These four-relator presentations can be readily used via simple extensions of Theorem 2.1 and Corollary 2.2 or Theorem 2.3 to give other three-relator presentations for $S_{4}(4)$ (by amalgamating power relations). We have tried to construct efficient presentations for $S_{4}(4)$ based on the presentations in Table 3 but have not succeeded.

\section{$4.11 U_{3}(5)$}

Even though $U_{3}(5)$ is the smallest simple group in our range, it is difficult to handle with our methods. Again, Methods 1 and 3 are hampered by the fact that $U_{3}(5)$ is not generated by any pair of elements of which one is an involution and the other has order 3 . Neither $U_{3}(5)$ nor its cover appeared in any of our censuses, and neither appeared as a one-relator quotient of any $C_{m} * C_{n}$. Indeed, we have not been able to find an efficient presentation for $\widehat{U}_{3}(5)$.

The group $U_{3}(5)$ has 9947 distinct generating pairs, and $\widehat{U}_{3}(5)$ has 88290 distinct generating pairs. We investigated all the distinct generating sets for $U_{3}(5)$ (in 13 CPU days) and found many efficient presentations for the group. Unfortunately, none was of a form suitable for applying Theorem 2.1, Corollary 2.2, or Theorem 2.3. There is a tantalizing collection of efficient presentations, and we list a selection of those we found in Table 4 . We have tried to construct efficient presentations for $\widehat{U}_{3}(5)$ based on these but have not succeeded.

It is worth noting that MAGMA presentations found are not necessarily shortest possible even in quite simple ways. The second presentation in Table 4 is readily converted by a simple Tietze transformation into presentations that are shorter by one. In Table 2 we tabulate a canonical representative of that presentation (produced by the ACME program [Havas and Ramsay 03b]).

The presentations listed in Table 4 were chosen because of their attractive structure or because coset enumeration is relatively easy. They include efficient presentations, and also some nice ones with one extra relator and that include three power relations.

These four-relator presentations can be readily used via simple extensions of Theorem 2.1, Corollary 2.2, or Theorem 2.3 to give three-relator presentations for $U_{3}(5)$ or its cover (by amalgamating power relations). Most of these came from applications of Method 2. However, the length-31 and length-37 presentations arose in a different way. Investigating $L_{3}(4)$ in [Campbell et al. 04], we found it useful to study one-relator quotients of the 


\begin{tabular}{|c|c|c|}
\hline Relators & Length & Cosets \\
\hline$a^{7}, B A B A B a b a b a, a b b a B a^{3} b^{3} A b a$ & 31 & 335319 \\
\hline$a^{7}, A b b a b A B^{3} a b, a b a a b b A B^{3} a a b$ & 31 & 353243 \\
\hline$b^{7}, a B a a b a B A^{3} b, A B B a^{3} b A A B B A B$ & 31 & 366504 \\
\hline$b^{7}, B a B A B a B A b A, b a B A b a^{3} b A B a b a$ & 31 & 1062315 \\
\hline$b^{5}, B A B A B a b A b A b a B A, B a B B A b b a b b A B B a$ & 33 & 3341611 \\
\hline$b^{8}, a^{3} b a^{3} b b a b b, a B a B^{3} a^{4} B^{3}$ & 33 & 13279158 \\
\hline$a^{7}, A b b A b a a B B a a b, A b b A A b b a a B B A^{3} B$ & 34 & 400830 \\
\hline$b^{7}, A B B A A B a a b a a B^{3}, A B a B^{3} a a B^{3} a B A$ & 35 & 1063840 \\
\hline$a^{4}, b A A B B A A B A B B a b A, b A A B A A B A A B A b^{3} a b A$ & 35 & 3200496 \\
\hline$b^{5}, B A B a B A B a B A b A B a, b A B A^{4} B A b a B a a B a$ & 35 & 4038434 \\
\hline$b^{4}, a a B a B B a a b b A B A b, a B B a B B A B B A b a^{5} B$ & 35 & 4268641 \\
\hline$b^{4}, B A b A B B a a b b a b a a, b A^{5} B A b b A b b a b b a$ & 35 & 4375702 \\
\hline$a^{7}, A A b b a^{3} b b A^{3} B B a B, A A b b A A b A^{3} B B A^{3} b$ & 39 & 1569322 \\
\hline 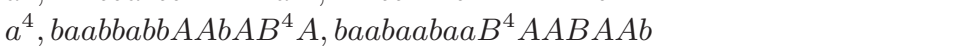 & 40 & 1608805 \\
\hline$b^{3} a B^{5} a, a a B a a B A^{5} B, A b b a^{3} B a B B a B^{3} A^{4} B$ & 41 & 1916333 \\
\hline$a^{3} b A^{4} b a, B a b b A^{3} B B a B, a b A b a a B a B a^{3} B B A A b A A b a B$ & 43 & 173182 \\
\hline$a b^{5} a b a B B a b, a^{3} B a^{3} B A^{5} B, a^{3} b a^{4} B^{3} A^{4} b$ & 43 & 329760 \\
\hline$a^{6} b A^{3} b a, b a^{5} b b a B^{4} a b, a a b a a B^{6} A^{3} b A b$ & 44 & 279893 \\
\hline$a b a^{3} b a b A A b$, BaabbaaBB $B A^{3} B, B a^{3} b^{3} a^{3} B B A^{7} B$ & 44 & 6335191 \\
\hline$a b^{5} a a B^{3} a, a^{3} B a^{3} B A^{4} B, A b^{6} a^{3} b^{4} A B B A A b$ & 45 & 4917429 \\
\hline$a^{7}, A b b A b A^{5} B B A^{5} b, A b b a^{5} b b A^{5} B B A^{3} B$ & 46 & 891029 \\
\hline$a^{3} B a^{3} B A^{5} B, b a^{3} b^{4} A^{5} b a, a a b^{5} a B^{3} A^{6} B$ & 47 & 382676 \\
\hline$b^{2}, A^{3} b A b a b a^{3} b a b a^{4} b A b, A b A b a b A b A b a^{3} b A b A b a b A b A b$ & 47 & 656937 \\
\hline$a^{3} b a^{3} b A^{4} b, B a^{4} b b a^{3} B A^{4} B, B a a b b a^{5} b^{4} a B B A B^{3} a$ & 51 & 14841176 \\
\hline$B A b^{4} a B^{6} A B, b a b b a b a B^{5} a B^{5} a, B A b A b^{7} a b A B^{3} A B B A B a B a$ & 59 & 1968195 \\
\hline$a^{3} B a^{3} B A^{5} B, A b a^{5} B a a b A A B A^{3} B A^{5} b, A b a a b a^{4} B a a b A A B A^{5} B A B A$ & 63 & 5962702 \\
\hline$a^{3} b a^{3} b A^{5} b, A b a^{5} B a a b A A B A^{6} b A^{3} B, A b a^{4} B a^{3} B a a b A b A^{5} b A^{3} B A$ & 66 & 8798157 \\
\hline$a a b A^{5} b a, b b a B a a B a a B a^{3} B^{5} A A b, b^{3}(a a B)^{4} a^{5}(b A A)^{5} b b$ & 68 & 2207159 \\
\hline$a^{5},(a b)^{3}, b^{7}$, aabaabaabAbbbAb & 31 & 1545569 \\
\hline$b^{3}, a^{7},(B a)^{5}, b a B A b a^{3} b A B a b a$ & 34 & 1512755 \\
\hline$(B a)^{3}, b^{7}, a^{8}, b b A^{4} B A B a^{4} b$ & 35 & 304591 \\
\hline$(a b)^{2}, a^{5}, b^{7}, a a b A A b b a B B a B^{3} A A B a B$ & 37 & 144589 \\
\hline$b^{7}, a^{8},(B A B)^{3}, B B A^{4} B a^{4} B B a$ & 38 & 839310 \\
\hline$a^{4},(B A)^{3},(B a)^{5}, B A A B A A B A b A b A B a a B a a B$ & 39 & 185439 \\
\hline$b^{3}, a^{8},(B a)^{7}, a B A^{4} b A A b a^{4} B a B$ & 42 & 625696 \\
\hline$a^{2}, b^{5},(a B)^{7}, a b a B a b b a b b a b b a B a b a b a b b a b$ & 47 & 155425 \\
\hline$b^{2}, a^{7},(A A b)^{5}, a a b A A b a^{3} b a^{3} b A A b a a b a b a b$ & 48 & 185983 \\
\hline
\end{tabular}

TABLE 4. Interesting presentations for $U_{3}(5)$.

group $(l, m, n)=\left\{a, b \mid a^{l}, b^{m},(a b)^{n}\right\}$. Those presentations are the shortest, best-enumerating instances of onerelator quotients of $(5,7,3)$ and $(5,7,2)$ that we found that give $U_{3}(5)$.

We investigated some of the distinct generating sets for the cover and failed to find any efficient presentations or any presentations to which Theorem 2.1, Corollary 2.2, or Theorem 2.3 could be applied. We did not find any moreattractive presentations than the ones we have listed for $U_{3}(5)$. As we have seen, there can be very many efficient presentations for a group. Once we start allowing extra relations, the number of presentations goes up very rapidly. In Table 5 we give a few of the 2-generator, 3 - relator presentations for $\widehat{U}_{3}(5)$ that we found, essentially as produced by our MAGMA program. We have been unable to use any of these to build an efficient presentation for $\widehat{U}_{3}(5)$.

We note that of the 24 presentations, all but 3 can be viewed as containing a relator that amalgamates two power relators (with not necessarily coprime orders). Indeed, the first presentation can be seen this way via two different amalgamations; its first relator may be obtained from $A^{5}(b a a)^{2}$ and its second relator from $(A A B)^{2} b^{7}$. If we replace any of the amalgamated relators in any of the presentations by the two underlying power relators, we obtain a presentation for $U_{3}(5)$. 


\begin{tabular}{|c|c|c|}
\hline Relators & Length & Cosets \\
\hline$b A A A b a a, b A A B A A b b b b b, B B a B A b A B A b b A b A b A B$ & 35 & 912849 \\
\hline$B B B a B A b b a B A, a a a a a a a b b b b b, B a a B A B a a B B B a B B$ & 37 & 474817 \\
\hline$A b a B B A b a b b b, a a a a a a a b b b b b, B a a B A B a a B B B a B B$ & 37 & 483482 \\
\hline$b A A A A b b b b, a B A B A b a a B a B A b a, b b a b a b A A b a B a b b a$ & 38 & 457952 \\
\hline$A A A A A b b b b, A b b a B A B A b b a B A b, B a B A A B A A B A B A b b A$ & 38 & 468011 \\
\hline$B A A A A B B B B, a b A b a B a a b a b a B a, b b a B a b A A b a b a b b a$ & 38 & 575437 \\
\hline$A b a B B A b a b b b, b a B B a a a b a b A b, A B A b A B a a a B A b A B A b$ & 39 & 484642 \\
\hline$B B B a B A b b a B A, b a B B a a a b a b A b, A B A b A B a a a B A b A B A b$ & 39 & 470694 \\
\hline$B A A A A B B B B, a b a B A B a a b a B a B a, b a B B B a B a a B A B a b b a$ & 39 & 549192 \\
\hline$B a B a B a b b b a, B B a B B A A B B a B B a, b a b A b a B a a b a a B a b A$ & 39 & 1180984 \\
\hline$A A A A A A A b b b b, a B a b a a a a b b A A A b, a B a b b A B A b b a B a B$ & 39 & 1135822 \\
\hline$b A A A A b b b b, A b a b A A b a b A B, b a B B a a B a B a a B B a b b A b A b$ & 40 & 476477 \\
\hline aаaаaaaaBBBB, aBBAAAAbbaBAB, $A B a b a B A A b b A A b b A$ & 40 & 649026 \\
\hline$A b A B a a a B a a b A, a b a B A A A B A b A b b, A B A B A B A B A b b b A B A B$ & 41 & 1149775 \\
\hline$B a B A B a a b A B B A, b A B A b b a a b a b a B a, B A B A B A B A b b b b b A$ & 41 & 616640 \\
\hline$A b A B a a a B a a b A, a b a B A A A B A b A B B, A B A B A B A B A b b b A B A B$ & 41 & 979135 \\
\hline$A B B B B A b A b A b, b a b a B a a a b b A B a b, b b A b A B a a a B a B B a b A$ & 41 & 768857 \\
\hline$A b b A B a a b A b a b, a b a B a B a a B B A b A B, A B B B B B B A b A b A b A b$ & 42 & 576893 \\
\hline$A b b A B B A b b A b b, a B A A A A A b b b a b b, a B A B A b A b a a b b a b a B a B$ & 43 & 716389 \\
\hline$B a b A A A A b a B a b a, b a a a a a a a b A b A b A, B A b a b a B a a B B A b b A B$ & 43 & 537103 \\
\hline$A b A b A b A b b b b b b, b b A B A b A A B a b b A A b, a a B a B A B a a B a B B a B B$ & 44 & 651280 \\
\hline$a B B a a a B B a b, B a B a a a a a B a B a B a, a B a B A B A A B A A b a B A A b b b A b$ & 45 & 537264 \\
\hline 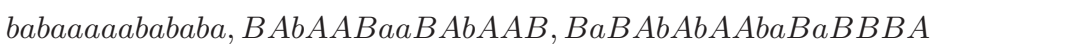 & 45 & 1031556 \\
\hline$B A b A b b A A b a B a B B B, B B a B a B a B a B B B B B B B, a b A B A b A A A b a B a b b A b$ & 48 & 999804 \\
\hline
\end{tabular}

TABLE 5. Some 3-relator presentations for $\widehat{U}_{3}(5)$.

\section{OPEN PROBLEMS}

In [Campbell et al. 04] we posed five open problems motivated by the investigation in that paper. The first three problems we presented there are as follows:

1. Is every simple group efficient? If not, which is the smallest inefficient simple group? Only one simple group of order less than one million is a candidate: $S_{4}(4)$. In particular, is $L_{2}\left(2^{n}\right)$ efficient for all $n$ ? Note that this has a positive solution for $n=2,3,4,5,6$.

2. Does the covering group of every finite simple group have a balanced presentation?

3. Is $A_{n}$ efficient for all $n$ ? This has a positive solution for $n \leq 9$. Even a much weaker question appears to be open. Is there a 2-generator presentation for $A_{n}$ with $k$ relators, where $k$ is independent of $n$ ?

There has been some progress on each of these. Question 3 asks whether $A_{n}$ is efficient for all $n$ and also poses a weaker question: is there a 2-generator presentation for $A_{n}$ with $k$ relators, where $k$ is independent of $n$ ? The weaker question has been answered in the affirmative in [Bray et al. 06, Guralnick et al. 07a, Guralnick et al. 07b]. In [Bray et al. 06], a 3-generator presentation is given with at most 246 relators, which, the authors note, may be used to obtain a 2-generator presentation with a bounded number of relations. In [Guralnick et al. 07a], a 27-generator presentation with at most 122 relators is given. The authors of [Guralnick et al. 07b] state that $A_{n}$ has a presentation with 4 generators and 10 relators. In fact, these papers, together with [Korchagina and Lubotzky 06], also give information on Question 1: is every finite simple group efficient? In [Guralnick et al. 07a] it is proved that every finite simple group (except perhaps the Ree groups) has a presentation with a bounded number of relators (certainly less than 500). Indeed, with respect to Question 2, [Wilson 06] states, "It seems reasonable to conjecture that the covering group of every finite simple group has a presentation with two generators and two relators."

On the one hand, our success in finding many efficient presentations supports this conjecture. On the other 
hand, our failure as yet to find efficient presentations for $\widehat{U}_{3}(5)$ and $S_{4}(4)$ reveals these as the only possible counterexamples of order less than one million.

\section{ACKNOWLEDGMENTS}

The second and third authors were partially supported by the Australian Research Council. The research was also partially supported by the Engineering and Physical Sciences Research Council grant EP/C523229/01, "Multidisciplinary Critical Mass in Computational Algebra and Applications."

\section{REFERENCES}

[Bosma et al. 97] Wieb Bosma, John Cannon, and Catherine Playoust. "The Magma Algebra System I: The User Language." J. Symbolic Comput. 24 (1997), 235-265.

[Bray et al. 06] J. N. Bray, M. D. E. Conder, C. R. LeedhamGreen, and E. A. O'Brien. "Short Presentations for Alternating and Symmetric Groups." Preprint, 2006.

[Campbell and Robertson 80] C. M. Campbell and E. F. Robertson." A Deficiency Zero Presentation for SL $(2, p)$." Bull. London Math. Soc. 12 (1980), 17-20.

[Campbell and Robertson 84] C. M. Campbell and E. F. Robertson. "Presentations for the Simple Groups $G, 10^{5}<$ $|G|<10^{6}$." Comm. Algebra 12 (1984), 2643-2663.

[Campbell and Robertson 88] Colin M. Campbell and Edmund F. Robertson. "Computing with Finite Simple Groups and Their Covering Groups." In Computers in Algebra, edited by M. C. Tangora, pp. 17-26. New York: Marcel Dekker, 1988.

[Campbell et al. 86] C. M. Campbell, E. F. Robertson, T. Kawamata, I. Miyamoto, and P. D. Williams. "Deficiency Zero Presentations for Certain Perfect Groups." Proc. Roy. Soc. Edinburgh 103A (1986), 63-71.

[Campbell et al. 89] Colin M. Campbell, E. F. Robertson, and P. D. Williams. "Efficient Presentations for Finite Simple Groups and Related Groups." In Groups-Korea 1988, pp. 65-72, Lecture Notes in Mathematics 1398. New York: Springer-Verlag, 1989.

[Campbell et al. 90] C. M. Campbell, E. F. Robertson, and P. D. Williams. "On Presentations of PSL $\left(2, p^{n}\right)$." J. Austral. Math. Soc. 48 (1990), 333-346.

[Campbell et al. 03] Colin M. Campbell, George Havas, Alexander Hulpke, and Edmund F. Robertson. "Efficient Simple Groups." Comm. Algebra 31 (2003), 5191-5197.

[Campbell et al. 04] Colin M. Campbell, George Havas, Colin Ramsay, and Edmund F. Robertson. "Nice Efficient Presentations for All Small Simple Groups and Their Covers." LMS J. Comput. Math. 7 (2004), 266-283.

[Conder et al. 06] Marston Conder, George Havas, and Colin Ramsay. "Efficient Presentations for the Mathieu Simple Group $M_{22}$ and Its Cover." In Finite Geometries, Groups, and Computation, pp. 33-42. Berlin: Walter de Gruyter, 2006.
[Conway et al. 85] J. H. Conway, R. T. Curtis, S. P. Norton, R. A. Parker, and R. A. Wilson. Atlas of Finite Groups. Oxford: Oxford University Press, 1985.

[Epstein 61] D. B. A. Epstein. "Finite Presentations of Groups and 3-Manifolds." Quart. J. Math. Oxford Ser. (2) 12 (1961), 205-212.

[Gamble et al. 04] Greg Gamble, Alexander Hulpke, George Havas, and Colin Ramsay. "GAP Package ACE; Advanced Coset Enumerator." Available online (http://www. gap-system.org/Packages/ace.html), 2004.

[GAP Group 04a] The GAP Group. "GAP-Groups, Algorithms, and Programming, Version 4.4." Available online (http://www.gap-system.org/), 2004.

[GAP Group 04b] The GAP Group. "Balanced Presentations for Covering Groups of Simple Groups." Available online (http://www.gap-system.org/Doc/Examples/ balanced.html), 2004.

[Guralnick et al. 07a] R. M. Guralnick, W. M. Kantor, M. Kassabov, and A. Lubotzky. "Presentations of Finite Simple Groups: A Quantitative Approach." To appear in J. Amer. Math. Soc. Preprint, 2007.

[Guralnick et al. 07b] R. M. Guralnick, W. M. Kantor, M. Kassabov, and A. Lubotzky. "Presentations of Finite Simple Groups: A Computational Approach." Preprint, 2007.

[Havas and Ramsay 00] George Havas and Colin Ramsay. "Proving a Group Trivial Made Easy: A Case Study in Coset Enumeration." Bull. Austral. Math. Soc. 62 (2000), $105-118$.

[Havas and Ramsay 01] George Havas and Colin Ramsay. "Coset Enumeration: ACE Version 3.001." Available online (http://www.itee.uq.edu.au/ havas/ace3001.tar.gz), 2001.

[Havas and Ramsay 03a] George Havas and Colin Ramsay. "Short Balanced Presentations of Perfect Groups." Groups St Andrews 2001 in Oxford, Volume 1, London Mathematical Society Lecture Note Series 304, pp. 238-243. Cambridge: Cambridge University Press, 2003.

[Havas and Ramsay 03b] George Havas and Colin Ramsay. "Breadth-First Search and the Andrews-Curtis Conjecture." Internat. J. Algebra Comput. 13 (2003), 61-68.

[Havas et al. 04] George Havas, M. F. Newman, and E. A. O'Brien. "On the Efficiency of Some Finite Groups." Comm. Algebra 32 (2004), 649-656.

[Jamali and Robertson 89] A. Jamali and E. F. Robertson. "Efficient Presentations for Certain Simple Groups." Comm. Algebra 17 (1989), 2521-2528.

[Korchagina and Lubotzky 06] Inna Korchagina and Alexander Lubotzky. "On Presentations and Second Cohomology of Some Finite Simple Groups." Publ. Math. Debrecen 69 (2006), 341-352.

[Sunday 72] J. G. Sunday. "Presentations of the Groups SL $(2, m)$ and PSL $(2, m)$." Canad. J. Math. 24 (1972), 1129-1131.

[Wilson 06] John S. Wilson. "Finite Axiomatization of Finite Soluble Groups." J. London Math. Soc. (2) 74 (2006), 566582. 
Colin M. Campbell, School of Mathematics and Statistics, University of St Andrews, North Haugh, St Andrews, Fife KY16 9SS, Scotland (cmc@st-andrews.ac.uk)

George Havas, ARC Centre for Complex Systems, School of Information Technology and Electrical Engineering, The University of Queensland, Queensland 4072, Australia (havas@itee.uq.edu.au)

Colin Ramsay, ARC Centre for Complex Systems, School of Information Technology and Electrical Engineering, The University of Queensland, Queensland 4072, Australia (cram@itee.uq.edu.au)

Edmund F. Robertson, School of Mathematics and Statistics, University of St Andrews, North Haugh, St Andrews, Fife KY16 9SS, Scotland (efr@st-andrews.ac.uk)

Received November 16, 2006; accepted December 13, 2006. 